\title{
Les médias et « l'objet swahili »
}

\section{Sylvie Capitan et Annie Lenoble-Bart}

\section{CpenEdition}

\section{Journals}

Édition électronique

URL : https://journals.openedition.org/eastafrica/592

DOI : 10.4000/eastafrica.592

ISSN : 2790-1076

\section{Éditeur}

IFRA - Institut Français de Recherche en Afrique

\section{Édition imprimée}

Date de publication : 1 septembre 2008

Pagination : $1-8$

ISSN : 2071-7245

\section{Référence électronique}

Sylvie Capitan et Annie Lenoble-Bart, «Les médias et « l'objet swahili » », Les Cahiers d'Afrique de l'Est / The East African Review [En ligne], 40 | 2008, mis en ligne le 07 mai 2019, consulté le 09 décembre 2021. URL : http://journals.openedition.org/eastafrica/592 ; DOI : https://doi.org/10.4000/eastafrica. 592

Ce document a été généré automatiquement le 9 décembre 2021.

Les Cahiers d'Afrique de l'Est / The East African Review 


\title{
Les médias et « l'objet swahili »
}

\author{
Sylvie Capitan et Annie Lenoble-Bart
}

1 Le programme ANR de recherche «Les dimensions de l'objet swahili » a intégré dès ses débuts la dimension médiatique. Conduit par des géographes et des linguistes, il s'inscrit néanmoins dans une interdisciplinarité associant sociologues, historiens et chercheurs en sciences de l'information et de la communication. Ces derniers ont développé une approche dont nous rendons compte. L'ANR étant à ses débuts, le présent article n'a pas pour ambition de délivrer des premiers résultats de recherche. Il se propose plus modestement de développer les problèmes épistémologiques que pose la question des médias et du swahili dans une perspective interdisciplinaire ainsi que d'annoncer les grandes lignes directrices qui seront suivies au cours de ce travail. Programme de recherche plus que résultat de recherche, il aborde néanmoins des questions importantes : les médias doivent-ils être abordés comme des outils ou comme les miroirs d'une réalité socio-culturelle? Les médias sont-ils seulement les vecteurs d'une langue ou bien aussi d'une «identité »? Que révèlent les médias de la position symbolique de la langue swahili?

\section{Contexte et pertinence d'une approche médiatique dans une recherche sur le swahili}

2 L'originalité du programme ANR est d'aborder le swahili sous une triple dimension, d'où le choix de son intitulé «L'objet swahili ». En effet, dans cette recherche, le swahili est abordé non seulement comme une langue - le kiswahili - mais aussi comme une identité, et enfin comme un objet potentiellement "cartographiable». L'un des objectifs est d'identifier et de cartographier les contours d'une éventuelle «aire swahili ». Cet objectif impose de réfléchir à ce que peut vouloir signifier cette notion: s'agit-il des territoires dans lequel le kiswahili est la langue principalement parlée, s'agit-il des territoires où le kiswahili est au moins compris ou s'agit-il plutôt des espaces où les individus se prévalent et se reconnaissent dans une identité swahili ? Cette « aire » est-elle une notion de chercheurs ou est-elle vécue comme telle sur le terrain et quels en sont ses marqueurs? Enfin, si cette aire existe, quels en sont ses 
contours, ses formes internes de structuration, sur quels réseaux s'appuie-t-elle ? C'est à ces questions que se propose de répondre l'ANR en capitalisant l'apport des géographes, des linguistes, des historiens et des chercheurs en information et communication.

3 L'angle adopté ici consiste à s'interroger sur l'espace médiatique kiswahili et cherche à savoir comment il s'articule à l'aire swahili. L'espace médiatique kiswahili se définit non par ses frontières - qui sont de toute évidence transnationales et globales - mais par les acteurs qui le constituent: les médias qui diffusent en kiswahili. L'intérêt de réfléchir aux liens qu'entretiennent les médias avec une langue africaine se justifie par l'originalité du kiswahili dans l'espace linguistique africain. En effet, le kiswahili est non seulement une langue africaine très répandue, mais aussi une des langues qui, pour des raisons historiques et politiques, justifient d'une des plus fortes légitimités intellectuelles. La Tanzanie l'a officiellement adoptée la première comme langue officielle; elle est langue d'enseignement; elle est langue de littérature dans toute l'Afrique de l'Est, langue aussi dans laquelle les élites n'hésitent pas à s'exprimer en public. Peu de langues africaines offrent une telle visibilité. Dans un tel contexte, il est intéressant de se demander comment les médias en usent. À quels publics destinent-ils ces programmes en kiswahili? Ces programmes visent-ils seulement les publics qui ne comprennent pas une autre langue, ou parviennent-ils à convaincre des publics qui ont le choix de se tourner vers des textes médiatiques anglophones ou d'une autre langue? Comment la légitimité du kiswahili s'exprime-t-elle à travers les médias : langue d'une minorité, langue de marqueur identitaire, langue d'information, langue de divertissement...

Pour aborder ces questions, cette recherche se propose de construire son travail autour de trois axes: cartographier l'espace médiatique du kiswahili, aborder son usage médiatique comme le miroir de sa position symbolique et enfin faire des textes médiatiques des sources de l'analyse linguistique avec comme question de fond, les médias sont-ils sources d'innovations linguistiques ou bien des outils de standardisation de la langue?

\section{Cartographier l'espace médiatique kiswahili}

$5 \quad$ Le premier objectif est de procéder au recensement des médias diffusant en kiswahili. Vaste entreprise qui entend concerner trois types de médias: les journaux, les télévisions et les radios. Les sites internet feront l'objet d'une méthodologie particulière qui ne sera pas abordée ici. Étant un média déterritorialisé, pour lequel les catégories pertinentes pour les médias de masse traditionnels font peu de sens (lieux d'émission, zone de couverture, audiences couvertes, etc.), ils seront abordés dans ce projet mais selon une méthodologie spécifique qui ne sera pas exposée dans cet article. Cependant, les trois autres médias de masse feront l'objet d'un recensement systématique selon un triple niveau d'analyse :

- Le niveau de la production. Quels sont les médias qui diffusent en kiswahili? Ce niveau permet d'introduire les médias internationaux qui diffusent en kiswahili depuis des zones non swahiliphones, comme la Deutsche Welle.

- Le niveau de la réception : où est-il possible de recevoir des programmes ou journaux en kiswahili? 
- Le niveau des audiences : tenter d'établir le niveau et la nature des audiences des médias utilisant le kiswahili. Ce niveau est sans doute le plus difficile à établir. Il n'est pas envisageable de procéder à des relevés d'audiences pour tous les médias recensés, il faudra sans doute recourir à des données de seconde main.

Ce recensement devra intégrer un certain nombre d'indicateurs pour être pertinents : les médias recensés diffusent-ils uniquement en kiswahili ou pratiquent-ils le pluralisme linguistique? Quelle est la proportion du kiswahili ? Quelle est la zone de couverture de ces médias? Toutes ces informations devraient pouvoir figurer sur une carte pour permettre de voir les lieux d'implantation de ces médias, leurs zones d'émissions, leurs liens avec les cartes linguistiques, leurs liens avec les zones dans lesquels le kiswahili est majoritaire ou minoritaire, etc.

\section{Les médias comme le miroir de la position symbolique du kiswahili}

7 L'élément caractéristique du kiswahili est d'être parlé et compris dans un grand nombre d'États. Pourtant, dans chacun d'entre eux ou même dans les différentes régions de ces pays, la position symbolique de cette langue diffère. Parfois langue de la majorité, parfois langue de la minorité, parfois langue de l'étranger, même de l'ennemi, parfois langue des populations autochtones, parfois enfin langue des élites ou langue des personnes non scolarisées... Elle occupe donc naturellement des positions symboliques différentes selon les contextes. Une des hypothèses de ce travail est de considérer que les médias peuvent se faire le reflet de ces réalités socio-culturelles diverses et peuvent aider à la compréhension de ces différentes représentations. En effet, l'usage que les médias font du kiswahili reflète en partie la manière dont on se représente cette langue et l'identité qui y est attachée. Quels sont les médias qui diffusent en kiswahili ? Font-ils de cette langue un marqueur de leur identité ? Quels sont les types de programmes diffusés : divertissement, information, sensibilisation? Quels sont les types des d'audiences visées? Quels types de médias usent de cette langue : les médias commerciaux, les médias communautaires, les journaux?

8 Afin de pouvoir répondre à ces questions, il s'agira de choisir quelques points privilégiés d'observation et d'identifier pour chacun d'eux :

- la carte linguistique de la région ;

- le paysage médiatique ;

- recenser les médias diffusant en kiswahili et pouvant être captés ;

- leur nature : publics, privés, nationaux, locaux, internationaux, religieux, communautaires, commerciaux...

- types de programmes diffusés : émissions d'appels téléphoniques, animations musicales, informations, publicités, émissions de sensibilisation... Faire l'analyse du format des textes médiatiques proposés en kiswahili.

Qui sont les personnes en charge de ces programmes, quel est leur lien avec cette langue, quelle est leur formation et quelles sont leur motivation pour diffuser dans cette langue?

Cet axe permettra de constater la position que le kiswahili occupe dans les médias et de la mettre en lien avec les études que géographes et linguistes auront faites dans les mêmes régions. 


\section{Les textes médiatiques comme des sources d'analyse linguistique}

11 Le troisième axe de l'étude renvoie à une analyse de contenu mais selon une perspective linguistique. En effet, les textes médiatiques ne constituent-ils pas des sources pertinentes pour suivre les différentes déclinaisons de la langue swahili? Malgré une volonté de standardisation, le kiswahili est parlé dans un très grand nombre de pays mais de manière diverse : le kiswahili de Lubumbashi diffère de celui de Mombassa, celui de Dar es Salaam de celui de Kigali... Il serait donc intéressant de relever des textes médiatiques, qu'ils soient écrits ou oraux, et de les analyser, avec l'appui des linguistes. Quel kiswahili parlent les médias? Comment ceux qui l'écrivent ou le parlent l'ont-ils appris? Quels sont les mots créés? Avec quelle autre langue le kiswahili est-il métissé ? Les médias sont-ils sources de création linguistique ou au contraire sont-ils les outils d'une certaine normalisation du langage?

Ces interrogations pourraient prendre appui sur différentes études de terrain. L'une d'entre elles pourrait consister par exemple à suivre la traduction à travers les médias de certaines notions comme celle de "démocratie» ou de "décentralisation". Comment les professionnels des médias procèdent-ils pour traduire ces notions : à quelle norme se réfèrent-ils?

13 Le tableau suivant synthétise les lignes directrices que ce programme de recherche, dans son volet médias, ambitionne de poursuivre.

\begin{tabular}{|c|c|c|}
\hline Objectifs & Méthodes & Résultats attendus \\
\hline $\begin{array}{l}\text { Cartographier l'espace } \\
\text { médiatique kiswahili }\end{array}$ & $\begin{array}{l}\text { Recenser les médias produisant en } \\
\text { kiswahili, recenser les zones où on peut } \\
\text { accéder à des sources médiatiques en } \\
\text { kiswahili, évaluer les audiences de ces } \\
\text { médias } \\
\text { Sources: enquêtes de terrain, revue de } \\
\text { littérature et documents des autorités } \\
\text { de régulation }\end{array}$ & $\begin{array}{l}\text { Visualiser la carte des } \\
\text { médias kiswahili } \\
\text { Préciser la proportion } \\
\text { d'usage du kiswahili et } \\
\text { indiquer les autres langues } \\
\text { utilisées } \\
\text { Comparer la " carte } \\
\text { médiatique » avec les cartes } \\
\text { linguistiques des pays }\end{array}$ \\
\hline $\begin{array}{l}\text { Définir la position } \\
\text { symbolique du swahili à } \\
\text { travers la manière dont les } \\
\text { médias usent du kiswahili }\end{array}$ & $\begin{array}{l}\text { Entreprendre des études de terrain } \\
\text { dans une perspective comparative : } \\
\text { Lubumbashi, la Rift Valley, Dar es } \\
\text { Salaam, Kampala, Kigali... }\end{array}$ & $\begin{array}{l}\text { Pour chacun des terrains, } \\
\text { etre à même de préciser: } \\
\text { - la place relative du } \\
\text { kiswahili dans la région } \\
\text { - le type de média qui } \\
\text { propose des programmes en } \\
\text { kiswahili } \\
\text { - les natures } \\
\text { programmes } \\
\text { - évaluer la nature des } \\
\text { audiences visées }\end{array}$ \\
\hline
\end{tabular}




\begin{tabular}{|l|l|l|}
\hline $\begin{array}{l}\text { Explorer le dynamisme } \\
\text { linguistique en analysant, } \\
\text { selon une perspective } \\
\text { linguistique, les textes } \\
\text { médiatiques kiswahili }\end{array}$ & $\begin{array}{l}\text { Constituer un corpus de textes } \\
\text { médiatiques à travers les régions } \\
\text { étudiées : articles, émissions de radio, } \\
\text { de télévision. S'attacher à la traduction } \\
\text { d'une même notion à travers les } \\
\text { médias de différentes régions }\end{array}$ & $\begin{array}{l}\text { lanalyse des linguistes } \\
\text { Définir si les médias sont } \\
\text { sources de création } \\
\text { linguistique ou au contraire } \\
\text { outils de standardisation du } \\
\text { langage. }\end{array}$ \\
\hline
\end{tabular}

\section{En guise de conclusion...}

Le programme annoncé est vaste. Il va nécessairement porter sur plusieurs États. Il va se concentrer sur l'Afrique de l'Est mais devra nécessairement prendre en compte des pays extérieurs à cette zone, les médias diffusant en kiswahili ne respectant pas les «frontières ».

Malgré cette ampleur, il espère pouvoir fournir des éléments d'information pertinents structurés autour des trois axes indiqués. Il s'inscrit, comme l'ensemble du projet, dans l'interdisciplinarité. Il est à considérer en l'état comme un cadre d'action dans lequel plusieurs questions transversales pourraient émerger : les médias, le kiswahili et la musique; les médias, le kiswahili et l'information; les médias, le kiswahili et la formation des journalistes ; les médias, le kiswahili et les autres langues vernaculaires ; les médias, le kiswahili et la standardisation du langage... Il devrait enfin enrichir un domaine de recherche assez peu exploré, que ce soit en Afrique de l'Est ou ailleurs : les médias africains et les langues. Dans quelles langues s'expriment les médias africains? Quelles sont les motivations de leurs choix linguistiques? Que révèle cet usage de la position symbolique respective des différentes langues et de leurs liens avec les élites? Cette étude pourrait apporter des débuts de réponses pour une des langues les plus parlées du continent. 